\title{
Evaluación de enfoques metodológicos que analizan la efectividad de las áreas naturales protegidas de Quintana Roo, México
}

\section{Resumen}

\author{
Luz del Alba Polanco Trujillo \\ Martha Angélica Gutiérrez Aguirre \\ Universidad de Quintana Roo \\ Unidad Académica Cozumel
}

Se analizan los enfoques metodológicos con los que se han evaluado metas concretas en las áreas naturales protegidas (ANP) de Quintana Roo, a la luz del desarrollo sustentable y considerando sus programas de manejo como los documentos rectores de sus actividades. Se parte de un planteamiento descriptivo de documentos institucionales y especializados, tanto nacionales como internacionales. El estado de Quintana Roo se concibe como la región con mayor biodiversidad registrada de México, pero también donde existen los desarrollos turísticos más importantes del país. De las 23 Anp decretadas para este estado, se estableció que 11 no cuentan con un programa de manejo. En la mayoría de las 12 que sí cuentan con él su evaluación se ha fundamentado en el cumplimiento de indicadores (administrativos, gobernabilidad, atributos del entorno biofísico, entre otros), bajo el enfoque de organizaciones sistémicas. Sin embargo, tal vez se deja de lado la opinión de las personas que habitan estas áreas, y probablemente se desaprovechan oportunidades de gobernanza que podrían hacer más efectivas a las ANP. Por último, se observó que no ha podido ser evaluada casi la mitad de ellas, ya que no cuentan con programa de manejo.

Palabras clave

Áreas naturales protegidas, gestión, gobernanza, efectividad.

Recibido: 13/05/2013 - Aceptado: 05/06/2013

Correo electrónico: luz.alba.pt@gmail.com 


\title{
Evaluation of methodological approaches used to analyze the effectiveness of protected nature areas in Quintana Roo, Mexico.
}

Luz del Alba Polanco Trujillo Martha Angélica Gutiérrez Aguirre

Universidad de Quintana Roo Unidad Académica Cozumel

\begin{abstract}
The methodological approaches with which concrete objectives have been evaluated in protected nature areas (ANP) of Quintana Roo state are analyzed. These were performed in the light of sustainable development and taking into consideration current management programs as the governing documents of their activities. The starting point is a descriptive exposition of institutional and specialized documents, both national and international. The state of Quintana Roo is conceived as the region with the highest biodiversity of Mexico and also with the most important tourist developments of the country. Of the 23 ANP's created by decrees for this state, it was established that eleven of them do not have a management program; assessment for the majority of the other twelve is based on mere compliance of indicators (administrative, governance, attributes of the biophysical environment, among others), under the systemic approach of organizations. However, possibly the opinion of the people that inhabit these areas is put aside, and probably opportunities are wasted for governance that could make ANp's more effective. Lastly, almost half have not been evaluated due to the lack of a management program.
\end{abstract}

KEY WORDS

Natural protected areas, effectiveness, management, governance. 


\section{Introducción}

En México, las primeras áreas naturales protegidas (ANP) se decretaron durante el gobierno del presidente Lázaro Cárdenas (1934-1940) (Elbers, 2011: 71). Vargas (1984: 177) señala que durante el periodo cardenista se impulsó de tal forma la creación de parques y reservas que se decretaron 82 áreas como parques nacionales y reservas forestales, con el propósito de conservar estos espacios en función del proyecto de crecimiento promovido por esa administración.

En el contexto mundial también se originó una preocupación creciente por los problemas ambientales y sociales, lo cual produjo que, incluso en las altas esferas de decisión, se discutieran temas como el desarrollo sustentable y se plantearan estrategias para la conservación. De acuerdo con Foladori (2002: 628), a partir de entonces se generó mayor conciencia sobre la responsabilidad social, económica, administrativa e incluso política hacia el medio ambiente; México fue partícipe de este impulso y, desde de la década de los sesenta hasta la actualidad, ha incrementado el número de áreas protegidas en el país.

En México, la creación y administración de estas áreas está definida en el artículo 3, fracción 1l, de la Ley General de Equilibrio Ecológico y Protección al Ambiente (LGEePA) (Dof, 1988: 2), como: "las zonas del territorio nacional y aquéllas sobre las que la nación ejerce su soberanía y jurisdicción, en donde los ambientes originales no han sido significativamente alterados por la actividad del ser humano o que requieren ser preservadas y restauradas".

El artículo 65 de la LGEEPA establece que una vez publicada la declaratoria de un ANP en el Diario Oficial de la Federación (DOF), la secretaría encargada tiene el plazo de un año para formular el programa de manejo (PM), asimismo, el director del área será el responsable de la ejecución y evaluación de este. Por su parte, en el artículo 77 del reglamento de la LGEEPA, en materia de ANP, se establece que: "El programa de manejo será revisado por lo menos cada cinco años, con el objeto de evaluar su efectividad y proponer posibles modificaciones". En atención a lo antes expuesto, este trabajo se enfocará en las metodologías que se han utilizado para evaluar la efectividad del manejo de las anp. 


\section{Acercamiento a los principios y operación de las áreas naturales protegidas de Quintana Roo}

En México se tienen decretadas 176 ANp, que cubren más de 25387972 hectáreas de su territorio. Es importante aclarar que $80 \%$ de las ANp se ubican en la superficie terrestre, mientras que $20 \%$ corresponde a las zonas marinas. La categoría más numerosa dentro de las ANP es la de los parques nacionales, y la más destacada como categoría de manejo y conservación son las reservas de la biósfera, que ocupan más de la mitad de la superficie protegida. ${ }^{1}$

En el estado de Quintana Roo existen -por decreto- 23 ANp, cuyas características generales se describen en el cuadro 1 .

\section{Cuadro 1. Descripción básica de las áreas naturales protegidas en Quintana Roo, México}

\begin{tabular}{|c|c|c|c|}
\hline NombRE & Categoría & $\begin{array}{l}\text { ¿Cuenta CON } \\
\text { PROGRAMA DE } \\
\text { MANEJO?* }^{*}\end{array}$ & $\begin{array}{l}\text { SUPERFICIE (HA) y } \\
\text { UBICACIÓN (MUNICIPIO) }\end{array}$ \\
\hline Uaymil & $\begin{array}{l}\text { Área de protección de } \\
\text { flora y fauna }\end{array}$ & No & $\begin{array}{l}84088 \\
\text { Felipe Carrillo Puerto }\end{array}$ \\
\hline Yum Balam & $\begin{array}{l}\text { Área de protección de } \\
\text { flora y fauna }\end{array}$ & No & $\begin{array}{l}313024 \\
\text { Lázaro Cárdenas }\end{array}$ \\
\hline San Felipe Bacalar & $\begin{array}{l}\text { Campo experimental } \\
\text { forestal }\end{array}$ & No & $\begin{array}{l}8000 \\
\text { Othón P. Blanco }\end{array}$ \\
\hline Banco Chinchorro & Reserva de la biósfera & Sí & $\begin{array}{l}144360 \\
\text { Othón P. Blanco }\end{array}$ \\
\hline Bala'an K'aax & $\begin{array}{l}\text { Área de protección de } \\
\text { flora y fauna }\end{array}$ & Sí & $\begin{array}{l}116480 \\
\text { Othón P. Blanco y José } \\
\text { Ma. Morelos }\end{array}$ \\
\hline Arrecifes de Xcalak & Parque nacional & Sí & $\begin{array}{l}13340 \\
\text { Othón P. Blanco }\end{array}$ \\
\hline
\end{tabular}


Cuadro 1. Descripción básica de las áreas naturales protegidas

en Quintana Roo, México

(Continuación)

\begin{tabular}{|c|c|c|c|}
\hline Nombre & Categoría & $\begin{array}{l}\text { ¿CuEnta CON } \\
\text { PROGRAMA DE } \\
\text { MANEJO?* }\end{array}$ & $\begin{array}{l}\text { SUPERFICIE (HA) Y } \\
\text { UBICACIÓN (MUNICIPIO) }\end{array}$ \\
\hline $\begin{array}{l}\text { Arrecifes de Puerto } \\
\text { Morelos }\end{array}$ & Parque nacional & Sí & $\begin{array}{l}9066 \\
\text { Benito Juárez y } \\
\text { Solidaridad }\end{array}$ \\
\hline $\begin{array}{l}\text { Otoch Ma'ax Yetel } \\
\text { Kooh }\end{array}$ & $\begin{array}{l}\text { Área de protección } \\
\text { de flora y fauna }\end{array}$ & Sí & $\begin{array}{l}5367.42 \\
\text { Lázaro Cárdenas y } \\
\text { Solidaridad }\end{array}$ \\
\hline $\begin{array}{l}\text { Manglares de Ni- } \\
\text { chupté }\end{array}$ & $\begin{array}{l}\text { Área de protección } \\
\text { de flora y fauna }\end{array}$ & No & $\begin{array}{l}4257 \\
\text { Benito Juárez }\end{array}$ \\
\hline El Edén & Privada & No & $\begin{array}{l}1492 \\
\text { Lázaro Cárdenas }\end{array}$ \\
\hline $\begin{array}{l}\text { Arrecifes de } \\
\text { Sian Ka’an }\end{array}$ & Reserva de la biósfera & Sí & $\begin{array}{l}34927 \\
\text { Solidaridad y Felipe } \\
\text { Carrillo Puerto }\end{array}$ \\
\hline Sian Ka'an & Reserva de la biósfera & Sí & $\begin{array}{l}528147 \\
\text { Solidaridad y Felipe } \\
\text { Carrillo Puerto }\end{array}$ \\
\hline $\begin{array}{l}\text { Arrecifes de } \\
\text { Cozumel }\end{array}$ & $\begin{array}{l}\text { Parque marino } \\
\text { nacional }\end{array}$ & Sí & $\begin{array}{l}11987 \\
\text { Cozumel }\end{array}$ \\
\hline $\begin{array}{l}\text { Costa Occidental de } \\
\text { Isla Mujeres Punta } \\
\text { Cancún y Punta } \\
\text { Nizuc }\end{array}$ & $\begin{array}{l}\text { Parque marino } \\
\text { nacional }\end{array}$ & Sí & $\begin{array}{l}8673.06 \\
\text { Benito Juárez e Isla } \\
\text { Mujeres }\end{array}$ \\
\hline $\begin{array}{l}\text { Parque Nacional } \\
\text { de Tulum }\end{array}$ & Parque nacional & No & $\begin{array}{l}664 \\
\text { Tulum }\end{array}$ \\
\hline $\begin{array}{l}\text { Reserva Especial } \\
\text { de la Biósfera Isla } \\
\text { Contoy }\end{array}$ & Parque nacional & Sí & $\begin{array}{l}5126 \\
\text { Isla Mujeres }\end{array}$ \\
\hline
\end{tabular}


Cuadro 1. Descripción básica de las áreas naturales protegidas en Quintana Roo, México

(Finaliza)

\begin{tabular}{|c|c|c|c|}
\hline Nombre & Categoría & $\begin{array}{l}\text { ¿CUENTA CON } \\
\text { PROGRAMA DE } \\
\text { MANEJO?* }\end{array}$ & $\begin{array}{l}\text { SUPERFICIE (HA) Y } \\
\text { UBICACIÓN (MUNICIPIO) }\end{array}$ \\
\hline $\begin{array}{l}\text { Santuario de la } \\
\text { Tortuga Marina } \\
\text { Xcacel-Xcacelito }\end{array}$ & $\begin{array}{l}\text { Zona sujeta a } \\
\text { conservación } \\
\text { ecológica }\end{array}$ & No & $\begin{array}{l}362.10 \\
\text { Tulum }\end{array}$ \\
\hline $\begin{array}{l}\text { Refugio Estatal de } \\
\text { Flora y Fauna Lagu- } \\
\text { na Colombia }\end{array}$ & $\begin{array}{l}\text { Zona sujeta a } \\
\text { conservación } \\
\text { ecológica }\end{array}$ & Sí & $\begin{array}{l}1113.54 \\
\text { Cozumel }\end{array}$ \\
\hline $\begin{array}{l}\text { Refugio Estatal } \\
\text { de Flora y Fauna } \\
\text { Sistema Lagunar } \\
\text { Chacmochuc }\end{array}$ & $\begin{array}{l}\text { Zona sujeta a } \\
\text { conservación } \\
\text { ecológica }\end{array}$ & No & $\begin{array}{l}1914.52 \\
\text { Benito Juárez e Isla } \\
\text { Mujeres }\end{array}$ \\
\hline $\begin{array}{l}\text { Refugio de Flora } \\
\text { y Fauna Laguna } \\
\text { Manatí }\end{array}$ & $\begin{array}{l}\text { Zona sujeta a conser- } \\
\text { vación ecológica }\end{array}$ & Sí & $\begin{array}{l}202.99 \\
\text { Benito Juárez }\end{array}$ \\
\hline $\begin{array}{l}\text { Santuario del } \\
\text { Manatí Bahía de } \\
\text { Chetumal }\end{array}$ & $\begin{array}{l}\text { Zona sujeta a } \\
\text { conservación } \\
\text { ecológica }\end{array}$ & No & $\begin{array}{l}281320 \\
\text { Othón P. Blanco }\end{array}$ \\
\hline Chankanaab & $\begin{array}{l}\text { Parque natural } \\
\text { municipal }\end{array}$ & No & $\begin{array}{l}9.23 \\
\text { Cozumel }\end{array}$ \\
\hline $\begin{array}{l}\text { Parque Urbano } \\
\text { Kaba }\end{array}$ & Parque urbano & No & $\begin{array}{l}41.48 \\
\text { Benito Juárez }\end{array}$ \\
\hline
\end{tabular}

Fuente: Elaboración propia a partir de Prezas (2011: 301). 
En la LGEEPA se establece que estas áreas, así como cualquiera que se encuentre en territorio mexicano, deben procurar los siguientes objetivos fundamentales:

- Preservar los recursos naturales de las distintas áreas biogeográficas del país

- Preservar la biodiversidad, sobre todo aquellas especies en peligro de extinción

- Asignar una zona donde se pueda realizar investigación científica

- Innovar tecnologías que logren conservar la biodiversidad

- Proteger las áreas naturales de otras áreas de gran afluencia cultural

Los objetivos básicos de las ANP se encuentran enmarcados tanto legal como institucionalmente, de modo que orientan las actividades de preservación y conservación de los recursos naturales, pero también las actividades productivas que las personas que las habitan realizan (Melo, 2002: 44). Algunas actividades productivas que tienen lugar en las ANP de Quintana Roo son la pesca, el aprovechamiento forestal y el turismo (Hernández et al., 2013: 74).

En México (y en el mundo) se han destinado Anp para fines turísticos y de recreación, así como para la investigación científica (Segrado, Arroyo y Amador, 2010: 85-86). Por la reconocida crisis ambiental que enfrentamos en la actualidad (Bruyere, Beh y Lelengula, 2009: 57; Elizalde, 2007: 6) y por la presión de grupos ambientalistas, ecologistas conservacionistas y de la sociedad en general, se ha intentado que actividades como el turismo en ANP creen nuevas estrategias de desarrollo con una visión sustentable (Palomino y López, 2008: 156). Por ello existe el turismo alternativo, en este caso en su segmento del ecoturismo, que, en teoría, al realizarse dentro o cerca de un ANp, debería convertirse en oportunidades de desarrollo económico, teniendo en cuenta el desarrollo humano, social y la preservación de los recursos naturales de los destinos turísticos (Palomino y López, 2008: 154). Esta premisa estaría acorde con los objetivos generales de un ANP. Pero ¿cómo analizar si en las ANP se fomenta una visión sustentable del turismo?

Una manera sencilla es asumir que la efectividad de cualquier anp depende del alcance de sus objetivos. Sin embargo, tal evaluación dependerá de la lupa 
con que se mire, y esto proporciona una ocasión para analizar desde qué enfoque metodológico se han evaluado los alcances de metas concretas establecidas en las anp de Quintana Roo, a la luz del desarrollo sustentable y considerando en particular las actividades turísticas que se llevan a cabo en ellas.

\section{Planteamientos metodológicos para abordar el estudio de las áreas naturales protegidas}

Los intentos por establecer una metodología homogénea que permita evaluar la efectividad de las ANP en México son muy recientes (Pomeroy et al., 2005: 497; 2006: 139). Sus evaluaciones se han dirigido hacia las actividades productivas que en estas se llevan a cabo, en procesos administrativos, atributos biofísicos de los ecosistemas, gobernabilidad y/o en el beneficio socioeconómico que traen a sus habitantes. En Quintana Roo, 80 \% de las anp que cuentan con programas de manejo ofrecen servicios turísticos entre otras actividades productivas (Prezas, 2011: 303-305).

En las áreas naturales protegidas es común usar indicadores para medir el desempeño con base en una visión sistémica y no jerárquica de las organizaciones, metodología que da importancia a procesos que fomentan una planificación estratégica (Arriagada, 2002: 42; Pomeroy et al., 2006: 137) y los concibe como generadores de relaciones sinérgicas (Pomeroy et al., 2005: 485) dentro de las áreas.

La teoría afirma que el uso de indicadores ha permitido hacer observaciones sistemáticas, estructuradas y secuenciales que suministran los datos necesarios para abordar los problemas y debilidades del manejo y tomar decisiones acertadas; esta información es fundamental para su gestión (Cifuentes, Izurieta y Henrique de Faria, 2000: 10; Leverington, Hockings y Lemos Costa, 2008: 6).

A continuación se enlistan algunas metodologías fundamentadas en el sistema de indicadores que se han utilizado en Anp de América Latina y el Caribe para evaluar su efectividad (cuadro 2). 
Cuadro 2. Metodología para evaluar las áreas naturales protegidas

\begin{tabular}{|c|c|c|c|}
\hline Metodología & Descripción & Propósito & Indicadores \\
\hline $\begin{array}{l}\text { RAPPAM (Rapid } \\
\text { Assessment and } \\
\text { Priorization of } \\
\text { Protected Areas } \\
\text { Management) } \\
\text { Evaluación } \\
\text { Rápida y } \\
\text { Priorización de la } \\
\text { Gestión de Áreas } \\
\text { Protegidas. }\end{array}$ & $\begin{array}{l}\text { Está diseñada para } \\
\text { las comparaciones } \\
\text { de } \\
\text { amplio nivel entre } \\
\text { varias AP. }\end{array}$ & $\begin{array}{l}\text { * Crear conciencia } \\
\text { y apoyo. } \\
\text { * Mejorar la gestión } \\
\text { (manejo adaptativo) } \\
\text { a nivel de sistemas. }\end{array}$ & $\begin{array}{l}\text { - Presiones y amenazas } \\
\text { - Biológicos } \\
\text { - Socioeconómicos } \\
\text { - Vulnerabilidad } \\
\text { - Objetivos } \\
\text { - Seguridad jurídica } \\
\text { - Diseño del sitio y la planeación } \\
\text { - Personal } \\
\text { - Comunicación e información } \\
\text { - Infraestructura } \\
\text { - Finanzas } \\
\text { - Administración (planificación) } \\
\text { - Administración (toma de decisio- } \\
\text { nes y prácticas) } \\
\text { - Investigación y monitoreo } \\
\text { - Salidas } \\
\text { - Sistema de áreas } \\
\text { - Políticas de la zona } \\
\text { - Políticas del medio ambiente }\end{array}$ \\
\hline $\begin{array}{l}\text { MPA (Marine } \\
\text { Protected Areas) } \\
\text { Mesoamericana. } \\
\text { Evaluación Rá- } \\
\text { pida de la Efecti- } \\
\text { vidad de Manejo } \\
\text { en Áreas Marinas } \\
\text { Protegidas (Aмp) } \\
\text { de Mesoamérica. }\end{array}$ & $\begin{array}{l}\text { Tiene un sistema } \\
\text { de puntuación } \\
\text { que mide indica- } \\
\text { dores; está di- } \\
\text { señada para ser } \\
\text { una herramienta } \\
\text { rápida, incluye } \\
\text { un marco para la } \\
\text { medición de los } \\
\text { resultados y la } \\
\text { integridad eco- } \\
\text { lógica; se utiliza } \\
\text { para medir la } \\
\text { efectividad de las } \\
\text { Amp. }\end{array}$ & $\begin{array}{l}\text { * Mejorar la gestión } \\
\text { (manejo adaptati- } \\
\text { vo). }\end{array}$ & $\begin{array}{l}\text { Socioeconómicos: } \\
\text { - Distribución del proceso for- } \\
\text { mal a la comunidad } \\
\text { - Participación de los interesa- } \\
\text { dos } \\
\text { - Prácticas e intensidad del uso } \\
\text { local de los usos marinos } \\
\text { - Empleo en función de los } \\
\text { recursos marinos } \\
\text { - Estado de la infraestructura de } \\
\text { servicio }\end{array}$ \\
\hline
\end{tabular}


Evaluación de enfoques metodológicos que analizan la efectividad de las áreas naturales protegidas

Cuadro 2. Metodología para evaluar las áreas naturales protegidas (Continuación)

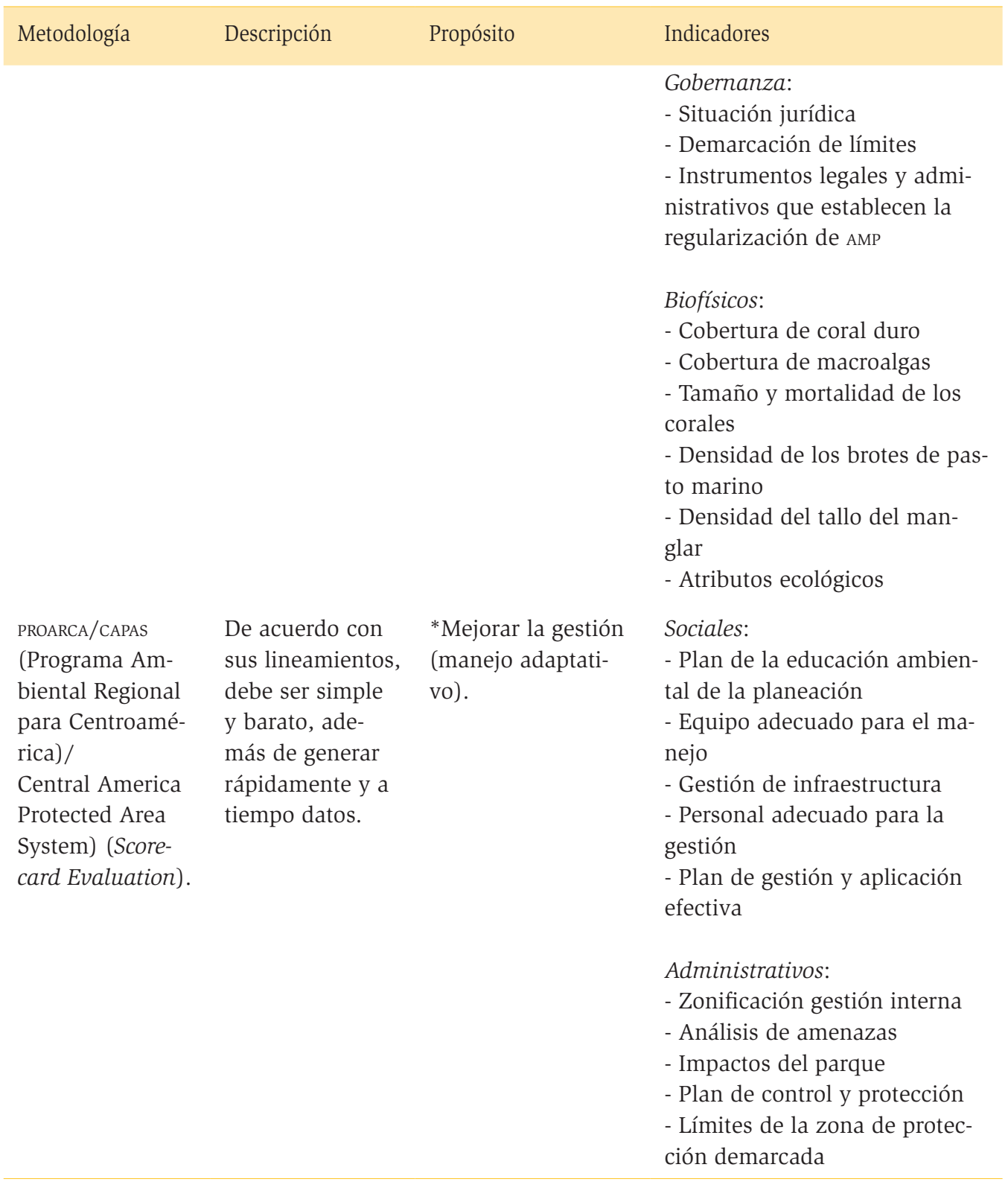


Cuadro 2. Metodología para evaluar las áreas naturales protegidas

(Continuación)

\begin{tabular}{|c|c|c|c|}
\hline Metodología & Descripción & Propósito & Indicadores \\
\hline $\begin{array}{l}\text { SIMEc (Sistema } \\
\text { de Información, } \\
\text { Monitoreo y } \\
\text { Evaluación para } \\
\text { la Conservación) } \\
\text { México. }\end{array}$ & $\begin{array}{l}\text { Es una evalua- } \\
\text { ción rápida con } \\
\text { base en un cues- } \\
\text { tionario score- } \\
\text { card, incluye } \\
\text { seis elementos } \\
\text { de gestión iden- } \\
\text { tificados en el } \\
\text { marco IUcNwcPA } \\
\text { (International } \\
\text { Union for Con- } \\
\text { servation of } \\
\text { Nature/World } \\
\text { Commission on } \\
\text { Protected Areas): }\end{array}$ & $\begin{array}{l}\text { * Mejorar la gestión } \\
\text { (manejo adaptati- } \\
\text { vo). } \\
{ }^{*} \text { Rendir cuentas/ } \\
\text { auditoría. }\end{array}$ & $\begin{array}{l}\text { Recursos (naturales y cultura- } \\
\text { les): } \\
\text { - Conectividad de la zona va- } \\
\text { luada y documentada } \\
\text { - Especies indicadoras identifi- } \\
\text { cadas y estudiadas } \\
\text { Política legal y económica: } \\
\text { - Aplicación de la ley } \\
\text { - La descentralización de la ad- } \\
\text { ministración del área protegida } \\
\text { Financieros: } \\
\text { - Plan para el financiamiento a } \\
\text { largo plazo de las Ap identifica- } \\
\text { das y valoradas } \\
\text { Procesos: } \\
\text { - Área total de la AP con los } \\
\text { certificados de conservación } \\
\text { - Número de entidades que } \\
\text { participan en el proyecto de } \\
\text { conservación } \\
\text { - La inversión de fuentes alter- } \\
\text { nas en el Ap } \\
\text { - Áreas de la región de conser- } \\
\text { vación prioritaria con la gestión } \\
\text { sostenible }\end{array}$ \\
\hline
\end{tabular}

continúa 
Cuadro 2. Metodología para evaluar las áreas naturales protegidas

\section{(Continuación)}

\begin{tabular}{|c|c|c|c|}
\hline Metodología & Descripción & Propósito & Indicadores \\
\hline $\begin{array}{l}\text { METT (Manage- } \\
\text { ment Effective- } \\
\text { ness Tracking } \\
\text { Tool) } \\
\text { Herramienta de } \\
\text { seguimiento de } \\
\text { la efectividad de } \\
\text { gestión. }\end{array}$ & $\begin{array}{l}\text { Capaz de propor- } \\
\text { cionar un siste- } \\
\text { ma de informa- } \\
\text { ción armonizado } \\
\text { de evaluación de } \\
\text { las Ap, adecuado } \\
\text { para la replica- } \\
\text { ción; incluye seis } \\
\text { elementos de } \\
\text { gestión: contex- } \\
\text { to, planificación, } \\
\text { insumos, proce- } \\
\text { sos, productos y } \\
\text { resultados. }\end{array}$ & $\begin{array}{l}\text { Mejorar la gestión } \\
\text { (manejo adaptativo) } \\
\text { y la rendición de } \\
\text { cuentas/auditoría. }\end{array}$ & $\begin{array}{l}\text { Proyecto: } \\
\text { - Número de eventos que con- } \\
\text { tribuyen a crear una cultura de } \\
\text { conservación } \\
\text { - AP programa de control y vi- } \\
\text { gilancia en coordinación con la } \\
\text { Profepa } \\
\text { - Recaudar fondos } \\
\text { - Desarrollo comercial y resi- } \\
\text { dente dentro de un AP } \\
\text { - La agricultura y acuicultura } \\
\text { dentro de un AP } \\
\text { - La producción y minería den- } \\
\text { tro de un AP } \\
\text { - Transporte y servicio de pasi- } \\
\text { llos dentro de un AP } \\
\text { - Utilización biológica de los } \\
\text { recursos } \\
\text { - Trastornos dentro de un AP } \\
\text { - Modificaciones en el sistema } \\
\text { natural } \\
\text { - Contaminación dentro de un } \\
\text { AP } \\
\text { - Eventos geológicos } \\
\text { - El cambio climático } \\
\text { - Amenazas culturales y socia- } \\
\text { les } \\
\text { - Régimen jurídico } \\
\text { - Cumplimiento de la ley } \\
\text { - Objetivos de las AP } \\
\text { - AP de diseño }\end{array}$ \\
\hline
\end{tabular}




\section{Cuadro 2. Metodología para evaluar las áreas naturales protegidas}

(Finaliza)

\begin{tabular}{|c|c|c|}
\hline Metodología & Descripción Propósito & Indicadores \\
\hline & & $\begin{array}{l}\text { - AP de marcación de límites } \\
\text { - Plan de gestión } \\
\text { - Proceso de planificación } \\
\text { - Sistema de protección } \\
\text { - Investigación } \\
\text { - Gestión de recursos } \\
\text { - Efectivos } \\
\text { - Formación de personal } \\
\text { - Presupuesto anual } \\
\text { - Gestión de presupuesto }\end{array}$ \\
\hline
\end{tabular}

Fuente: Elaboración propia con base en Leverington, Hockings y Lemos Costa (2008: 87-135).

\section{Áreas naturales, gestión y gobernanza}

El enfoque sistémico no es el único que evalúa los alcances de las metas en una organización y esta sección se aprovechará para detallar algunos otros. Una vez decretadas las ANP se presentan dilemas en cuanto a la determinación de su eficiencia, debido a que son bastante complejas: sus categorías y ubicación (cuadro 1) implican diferencias de uso; la existencia o carencia de programas de manejo origina retos heterogéneos aun en áreas del mismo tipo; la diversidad de características de sus habitantes produce diversidad en los sistemas productivos que se desarrollan dentro o en relación con el área, y los planes de ordenamiento territorial y programas de manejo generan que en una sola área convivan personas con diferentes intereses (Jamal y Stronza, 2009: 171). En consecuencia, en un ANP es difícil alcanzar y mantener un estado de alta eficiencia evitando ser solo un área geográfica establecida por decreto y con objetivos bien intencionados. Según Bruyere, Beh y Lelengula (2009: 49), la Comisión Mundial de Ambiente y Desarrollo y la Unión de Conservación Mundial han señalado la necesidad de la participación de las comunidades locales en los procesos de toma de decisiones en relación con el manejo y uso de las Anp. Esto conlleva aplicar en la realidad lo que en 
el plano teórico se conoce como gobernanza o autogobernanza, que se ha definido como una "interacción de factores (públicos y privados), unidos para resolver un problema y generar oportunidades sociales” (Kooiman y Bavinck, 2005: 12).

Además, y parafraseando a otros autores, el turismo sustentable en países en vía de desarrollo puede garantizar beneficios locales (económicos, de conservación, de calidad de vida, entre otros) creando oportunidades de legítima representatividad de las comunidades (Kiss, 2004: 232; Scheyvens, 1999: 248) en aspectos tan complejos como el uso y el manejo del ANP en cuestión.

En algunas ANP, cuyas actividades productivas se relacionan con el turismo, se ha sugerido que su gestión exitosa está fuertemente sustentada en la capacidad de los responsables o los líderes para integrar en su manejo a las comunidades locales y a todas las partes interesadas en la gestión (Sosa-Cordero, Liceaga-Correa y Seijo, 2008: 151). Para ello es fundamental la comunicación eficaz y eficiente entre grupos, la detección de las diferencias y coincidencias entre las comunidades y las partes involucradas, la participación de estas partes para la toma de decisiones y el alcance de metas comunes (Liu, Ouyang y Miao, 2010: 2260). Todas estas actividades legitiman el proceso de gestión y manejo de las ANp (Romagosa, Eagles y Duitschaever, 2012: 148).

Por lo tanto, es preciso entender que la participación local es vista como el factor clave que ha permitido y ayudado a los gerentes de las ANP a alcanzar sus metas. Lo anterior ya fue demostrado en Ecuador (Becker et al., 2005: 2703); México (Curtin, 2002: 883) y Nepal (Mehta y Heinen, 2001: 175).

Bruyere, Beh y Lelengula (2009: 50) afirman que la participación de las comunidades locales en la gestión de ANp provoca (como efecto benéfico colateral) que estas sean más valoradas y apreciadas y que, subsecuentemente, el turismo sustentable dentro de ellas sea considerado como una extraordinaria estrategia de conservación en las mismas.

En este sentido, Eagles, McCool y Haynes (2002: 113) afirman que el turismo sustentable es aquel que provee oportunidades de generar ventajas económicas para las partes interesadas, así como acciones de protección eficaces y eficientes para la protección y preservación del patrimonio natural y cultural, y el mejoramiento de la calidad de vida de las comunidades locales. 
El crecimiento a partir de la comunicación entre personas, la valoración de los procesos, el conocimiento de los involucrados y la rendición de cuentas, usando mecanismos de control y evaluación, han sido mencionados y están plenamente documentados como el primer paso para el éxito de las iniciativas de gestión y la ejecución de los planes de manejo y programas de turismo de naturaleza que se desarrollan en las ANP (Liu, Ouyang y Miao, 2010: 2256; Romagosa, Eagles y Duitschaever, 2012: 141).

Para el caso de México, una herramienta sobre la cual se fundamenta la gobernanza de cualquier ANP es el programa de conservación y manejo (PCM). Por ejemplo, según sus administradores, el PCM del Parque Nacional Arrecifes de Cozumel se elaboró teniendo en cuenta a servidores turísticos, investigadores, académicos y funcionarios públicos de los gobiernos federal, municipal y estatal (comunicación personal de C. A. González, 6 de abril de 2013).

A decir de sus administradores, las actividades de índole turística, como el número de buzos que se reciben en los arrecifes de Cozumel (una de las principales actividades productivas desarrolladas en la isla), se rigen por lo establecido en el PCM del parque, en el cual se precisan sistemas y mecanismos de control con el objeto de preservar la integridad del hábitat.

\section{Análisis de la efectividad de las ANP de Quintana Roo}

Del total de las anp que existen en Quintana Roo, únicamente 12 cuentan con planes de manejo (cuadro 1) y solo algunas de estas han sido evaluadas en cuanto a la efectividad de su manejo (cuadro 3), utilizando una metodología internacional (la MPA), la cual tiene indicadores clasificados en tres enfoques (socioeconómico, gobernabilidad y componente biofísico).

Es pertinente señalar que en las evaluaciones piloto de estas ANP no se consideraron todos los indicadores que integran cada enfoque de la metodología antes señalada. 
Cuadro 3. Áreas evaluadas de Quintana Roo

\begin{tabular}{|c|c|c|}
\hline ÁREAS PROTEGIDAS & $\begin{array}{l}\text { OBJETIVO DE LA } \\
\text { EVALUACIÓN }\end{array}$ & ENFOQUE METODOLÓGICO \\
\hline $\begin{array}{l}\text { - Reserva de la Biósfera } \\
\text { Banco Chinchorro } \\
\text { - Reserva de la Biósfera } \\
\text { Sian Ka'an } \\
\text { - Arrecifes de Sian Ka'an } \\
\text { - Parque Nacional Arreci- } \\
\text { fes de Cozumel } \\
\text { - Refugio Estatal de Flora } \\
\text { y Fauna Laguna Colom- } \\
\text { bia } \\
\text { - Arrecifes de Puerto Mo- } \\
\text { relos } \\
\text { - Parque Nacional Arreci- } \\
\text { fes de Xcalak } \\
\text { - Área de Protección de } \\
\text { Flora y Fauna Yum Ba- } \\
\text { lam } \\
\text { - Parque Nacional Isla } \\
\text { Contoy } \\
\text { - Zona Sujeta a Conser- } \\
\text { vación Ecológica Lagu- } \\
\text { na Manatí }\end{array}$ & $\begin{array}{l}\text { Evaluar la efectividad } \\
\text { de las áreas marinas } \\
\text { protegidas, hacia un } \\
\text { manejo adaptativo }\end{array}$ & $\begin{array}{l}\text { MPA Mesoamericana } \\
\text { (Evaluación Rápida de la } \\
\text { Efectividad de Manejo en } \\
\text { AMP de Mesoamérica) }\end{array}$ \\
\hline
\end{tabular}

Fuente: Elaboración propia con base en Lara-López (s.f.), Pomeroy, Parks y Watson (2006: 206), CepedaGonzález et al. (2009: 17).

El resultado de estas evaluaciones en relación con los indicadores ambientales es el incremento de alrededor de $200 \%$ en el número de especies detectadas en el área en comparación con el programa de manejo original; pero esto no necesariamente se debe a que las acciones de conservación hayan sido o sean efectivas, sino a que, en general, en los programas originales solo se enlistaron las especies que en ese momento se consideraban representativas (Reyes-Bonilla, 2011: 31). No obstante, y por fortuna, eventualmente se está alcanzando un conocimiento real de la biodiversidad de cada ANP, lo cual debe tomarse en cuenta en las actualizaciones de los programas de manejo. 
En cuanto a los componentes socioeconómicos y de gobernabilidad, es interesante observar que las evaluaciones incluyen indicadores exitosos siempre y cuando se incremente la cobertura (por ejemplo, en el número de pesquerías con ganancias económicas) y la focalización de la población (Cepeda-González et al., 2009: 59); por desgracia la opinión y experiencia de los propios pobladores casi no se incorpora.

En materia de efectividad de las Anp, el esfuerzo se ha centrado en acciones de protección, restauración, cultura y gestión de las mismas (Anónimo, 2012: 12), determinadas exclusivamente a través de indicadores.

Existen algunos programas tangenciales a los programas de manejo de las ANP cuyo objetivo es fomentar el desarrollo sustentable dentro de las áreas protegidas, como el Programa de Conservación para el Desarrollo Sostenible (Procodes) o las redes comunitarias de conservación. La efectividad de estos programas -subsidiados por el gobierno federal- se ha enfocado en determinar en qué medida se beneficia la población para la cual son diseñados -de nuevo mediante indicadores de cobertura y focalización-, según criterios de elegibilidad establecidos por la Comisión Nacional de Áreas Naturales Protegidas (Conanp), instancia responsable de la administración y manejo de las áreas protegidas en México (Sánchez-García et al., 2009).

Por otro lado, de acuerdo con la Conanp, existen algunas anp de Quintana Roo (cuadro 4) que cuentan con programas de monitoreo para especies o grupos de especies, que se basan en la metodología simec, pero no hay información de que se haya efectuado ya su evaluación; la única que se encuentra disponible en línea es la realizada en la Reserva de la Biósfera Isla San Pedro Mártir del estado de Sonora.

En el caso del Parque Nacional Arrecifes de Cozumel se realizan evaluaciones trimestrales y anuales con base en su programa operativo anual (POA), asimismo, dos veces al año se monitorean los impactos causados a los arrecifes por el turismo (comunicación personal de C. A. González, 6 de abril de 2013).

La poca incidencia en la evaluación de las Anp de Quintana Roo tal vez se debe a la falta de personal suficiente y/o suficientemente capacitado para practicar las evaluaciones, o a que no se dispone del presupuesto adecuado para adoptar alguna de las metodologías existentes, tal como se ha documentado en diversas partes de Latinoamérica (Pomeroy et al., 2006: 137), lo que resulta preocupante pues se sabe poco de la efectividad de manejo de cada área. 


\section{Cuadro 4. Áreas con programa de monitoreo}

Áreas marinas protegidas Objetivo de la evaluación Enfoque metodológico

- Parque Nacional Costa Occi- Realizar monitoreo a lar- Sistema de Información, dental de Isla Mujeres, Punta go plazo e incluir compo- Monitoreo y Evaluación Cancún y Punta Nizuc

- Arrecifes de Cozumel

- Reserva de la Biósfera Arrecifes de Sian Ka’an

- Parque Nacional Arrecifes

Alacranes

- Parque Nacional Isla Contoy

- Reserva de la Biósfera Ría

Lagartos

- Reserva de la Biósfera Sian

Ka'an

- Área de Protección de Flora

y Fauna Yum Balam

Fuente: Elaboración propia con base en información de la Conanp <www.conanp.gob.mx>.

El empleo de los indicadores usados en la evaluación de las áreas es importante, pero en general las personas que habitan y usan las ANP se sienten poco involucradas en las decisiones, el establecimiento de las metas y la definición de los propios indicadores (Pomeroy et al., 2006: 135), por lo que la probabilidad de generar oportunidades sociales disminuye. Debe transitarse de la medición de datos precisos con el uso exclusivo de indicadores hacia esquemas que incluyan relaciones hombre-comunidad-naturaleza, como el propuesto por Nahmad (2000: 21). Las acciones que permiten la participación de la sociedad fomentan que los usuarios del territorio hagan suyos los proyectos productivos (sean cuales sean), mientras que sus actividades cotidianas y culturales disminuyen el impacto sobre el medio ambiente, incluso blindando o reduciendo la dependencia económica de las áreas (para que estas sean efectivas). Ejemplo de este tipo de propuestas en Quintana Roo podrían ser las agrupaciones pesqueras que laboran dentro de la Reserva de la Biósfera de Sian Ka’an, cuya capacidad de organización, fundamentada en el esquema ejidal y de cooperativas, así como 
en una rigurosa legislación interna, ha conducido a una pesquería sustentable de langosta espinosa en la región central de Quintana Roo, documentada en varias oportunidades (Sosa-Cordero, Liceaga-Correa y Seijo, 2008: 149).

Es un hecho que aquellas áreas con decreto pero sin planes de manejo producen más preocupación: las 23 ANp de Quintana Roo ocupan una superficie territorial de 1573962.34 hectáreas del estado; sin embargo, en 44 \% de esta superficie no se han elaborado planes de manejo (cuadro 1); de tal forma que la eficiencia de casi la mitad de la superficie natural protegida del estado no puede ser evaluada porque, aunque están decretados, no han definido objetivos específicos ni metas concretas por alcanzar.

No obstante, han surgido iniciativas de la sociedad civil que, en colaboración con la Conanp, han determinado esquemas público-privados para obtener financiamiento que se invierte en programas de protección, investigación y gobernanza en algunas áreas protegidas de Quintana Roo.

Organizaciones civiles como estas son el Fondo Mexicano para la Conservación de la Naturaleza, ${ }^{2}$ los Miembros de Yum Balam o los Amigos de Kaba. Estas asociaciones han logrado obtener fondos, sobre todo de procedencia extranjera, como el Global Environmental Found, para operar las áreas naturales (Rosenzweig, 2004: 1). Con todo, sigue siendo materia pendiente la forma en que será evaluada la efectividad de estas superficies.

Además, algunas de las anp de Quintana Roo que no cuentan con PM se decretaron hace 30 años (por medio del Decreto del Parque Natural Municipal Chankanaab, por ejemplo), y conservarlas como tal comienza a dificultarse ante la presión de concesionarlas para realizar otras actividades (Prezas, 2011: 302). Un caso representativo al respecto es el Parque Urbano Kaba, al sur de la ciudad de Cancún, que en 2008 fue defendido por la ciudadanía con objeto de impedir que se concesionara para construir un estacionamiento (Varillas, 2008).

\section{Conclusión}

Casi la mitad de las Anp decretadas en Quintana Roo carecen de PM y, por lo tanto, es difícil saber si en realidad están cumpliendo los objetivos para los que

${ }^{2}<$ http://www.fmcn.org $>$. 
fueron creadas, lo que a su vez potencia el riesgo de perder superficie protegida. Existen casos documentados en los cuales las anp (tanto en el mundo como en Quintana Roo) han generado y evalúan su efectividad de una manera participativa, uno de ellos es el complejo Sian Ka'an, en Quintana Roo. Sin duda, este tipo de administración que involucra a la gente provoca que las ANP sean más valoradas y que cualquier actividad productiva realizada en ellas (incluida el turismo) genere oportunidades de desarrollo sustentable.

La evaluación de la efectividad de la mayoría de las AnP de Quintana Roo se ha efectuado bajo el enfoque metodológico MPA, que considera esencial el manejo adaptativo. Este enfoque se considera informativo y se ha usado también en otras regiones protegidas de Latinoamérica; sin embargo, en las áreas protegidas de la entidad se ha puesto demasiado énfasis en la evaluación de la organización desde un punto de vista sistémico, así como en el monitoreo de indicadores enfocados en la cobertura poblacional, lo que se traduce en una intensa atención hacia el carácter administrativo de las ANP en el estado, pero no se atiende en su justa dimensión la opinión de las personas que habitan estas áreas geográficas y probablemente se desaprovechan oportunidades de gobernanza que podrían hacer más efectivas a las áreas naturales protegidas de Quintana Roo.

\section{Fuentes consultadas}

Anónimo (2012) Estrategia Nacional para un desarrollo sustentable del turismo y la recreación en las áreas protegidas de México. Comisión Nacional de Áreas Naturales Protegidas (Conanp), México, 77 p.

Arriagada, R. (2002). Diseño de un sistema de medición de desempeño para evaluar la gestión municipal: Una perspectiva metodológica. Santiago de Chile: Instituto Latinoamericano y del Caribe de Planificación Económica y Social (ILPES)/Comisión Económica para América Latina y el Caribe (CEPAL), $188 \mathrm{pp}$.

Becker, C.D. et al. (2005). "Community-Based Monitoring of Fog Capture and Biodiversity at Loma Alta, Ecuador Enhance Social Capital and Institutional Cooperation”. Biodiversity and Conservation, 14 (11), 2695-2707.

Bruyere, L., A. Beh y G. Lelengula (2009). "Differences in Perceptions of Com- 
munication, Tourism Benefits and Management Issues in a Protected Area of Rural Kenya”. Environmental Management, 43 (1), 49-59.

Cepeda-González, M.F.M. et al. (comps.) (2009). Planeación para la conservación de la Reserva de la Biósfera de Banco Chinchorro: un esfuerzo conjunto. Mérida: The Nature Conservancy/Comisión Nacional de Áreas Naturales Protegidas (Conanp)/Amigos de Sian Ka'an/United States Agency for International Development, $91 \mathrm{pp}$.

Cifuentes, M., A. Izurieta y E. Henrique de Faria (2000). Medición de la efectividad del manejo de áreas protegidas. Turrialba: Unión Internacional para la Conservación de la Naturaleza (UICN)/Fondo Mundial para la Naturaleza (wwF)/German Technical Cooperation (Gтz) (Serie Técnica, 2), $108 \mathrm{pp}$.

Curtin, Ch.G. (2002). "Integration of Science and Community-Based Conservation in the Mexico/U.S. Borderlands". Conservation Biology, 16 (4), 880-886.

Dof (1988). Ley General de Equilibrio Ecológico y Protección al Ambiente. Diario Oficial de la Federación, 7 de junio.

------ (2000). Reglamento de la Ley General del Equilibrio Ecológico y la Protección al Ambiente en Materia de Áreas Naturales Protegidas. Diario Oficial de la Federación, 30 de noviembre.

Eagles, P.F.J., S.F. McCool y C.D. Haynes (2002). Sustainable Tourism in Protected Areas: Guidelines for Planning and Management. Gland/Cambridge: World Commission on Protected Areas (WCPA)/uICN/Natural Resources, $183 \mathrm{pp}$.

Elizalde, A. (2007). "Reseña de El desarrollo sostenible. Su dimensión ambiental y educativa". Polis. Revista de la Universidad Boliviana, 5, 5-16.

Elbers, J. (ed.) (2011). Las áreas naturales protegidas de América Latina: situación actual y perspectiva para el futuro. Quito: UICN.

Foladori, G. (2002). "Avances y límites de la sustentabilidad social”. Economía, Sociedad y Territorio, III (12), 621-637.

Hernández, J. et al. (2013). "Instituciones locales y procesos organizativos: el caso de la Reserva de la Biósfera de Sian Ka’an”. Estudios Sociales: Revista de Investigación Científica, 21 (41), 66-93. 
Jamal, T. y A. Stronza (2009). “Collaboration Theory and Tourism Practice in Protected Areas: Stakeholders, Structuring and Sustainability”. Journal of Sustainable Tourism, 17 (2), 169-189.

Kiss, A. (2004). "Is Community-Based Ecotourism a Good Use of Biodiversity Conservation Funds?" Trends in Ecology \& Evolution, 19 (5), 232-237.

Kooiman, J. y M. Bavinck (2005). "The Governance Perspective”, en J. Kooiman et al. (eds.). Fish for Life. Ámsterdam: University of Amsterdam Press, 11-24.

Lara-López O.F. (s.f.). Línea base regional sobre la efectividad de manejo en áreas marinas protegidas del Sistema Arrecifal Mesoamericano. Proyecto para la conservación y uso sostenible del Sistema Arrecifal Mesoamericano [en línea], 10 pp. Disponible en: http://www.scribd.com/ doc/55917583/Linea-Base-Regional-sobre-la-Efectividad-de-Manejoen-Areas-Marinas-Protegidas-del-Sistema-Arrecifal-Mesoamericano

Leverington, F., M. Hockings y K. Lemos Costa (2008). Management Effectiveness Evaluation in Protected Areas: Report for the Project "Global Study into Management Effectiveness Evaluation of Protected Areas". Gatton: The University of Queensland/uICN/wCPA/The Nature Conservancy/ WWF.

Liu, J., Z. Ouyang y H. Miao (2010). “Environmental Attitudes of Stakeholders and their Perceptions Regarding Protected Area-Community Conflicts: A Case Study in China”. Journal of Environmental Management, 91 (11), 2254-2262.

Mehta, J.N. y J.T. Heinen (2001). “Does Community-Based Conservation Shape Favorable Attitudes Among Locals? An Empirical Study from Nepal”. Environmental Management, 28 (2), 165-177.

Melo, C. (2002). Áreas naturales protegidas de México en el siglo xx. México: Universidad Nacional Autónoma de México.

Nahmad, S. (2000). "El Proyecto del Fondo Mundial para la Protección del Medio Ambiente (Gef) en cuatro áreas naturales protegidas de México y su impacto social”. Journal of Political Ecology: Case Studies in History and Society, 7, 19-42.

Palomino, B. y G. López (2008). "Políticas públicas y ecoturismo indígena en México”, en A. Palafox y O. Frausto. Turismo. Desastres naturales, sociedad y medio ambiente. Cozumel: Universidad de Quintana Roo, 149-168. 
Pomeroy, R.S., J.E. Parks y L.M. Watson (2006). Cómo evaluar una AmP. Manual de indicadores naturales y sociales para evaluar la efectividad de la gestión de Áreas Marinas Protegidas. Gland/Cambridge: uicn, 216 pp.

Pomeroy, R.S. et al. (2005). "How is your MPA doing? A Methodology for Evaluating the Management Effectiveness of Marine Protected Areas". Ocean \& Coastal Management, 48 (7), 485-502.

(2006). "Introducción a una metodología para evaluar la efectividad de la gestión de áreas marinas protegidas”, en I. Pisanty y M. Caso (comps.). Especies, espacios y riesgos. Monitoreo para la conservación de la biodiversidad. México: Secretaría de Medio Ambiente y Recursos Naturales/ Instituto Nacional de Ecología/Comisión para la Cooperación Ambiental/Unidos para la Conservación, 135-158.

Prezas, B. (2011). “Áreas naturales protegidas en Quintana Roo”, en C. Pozo, N. Armijo Canto y S. Calmé (eds.). Riqueza biológica de Quintana Roo. Un análisis para su conservación t. 1. México: El Colegio de la Frontera Sur/Comisión Nacional para el Conocimiento y Uso de la Biodiversidad/Gobierno del Estado de Quintana Roo/Programa de Pequeñas Donaciones, 300-309.

Reyes-Bonilla, H. (2011). Informe final del proyecto DM007. Monitoreos complementarios de algas, invertebrados y peces en el Parque Nacional Arrecifes de Cozumel. México: Universidad Autónoma de Baja California Sur, 69 pp.

Rivera, M. (2011). "Estado de la investigación evaluativa en el caso de las Áreas Marinas Protegidas de México”. CICIMAR Oceánides, 26 (2) pp. 9-17.

Romagosa, F., P.F.J. Eagles y W.B. Duitschaever (2012). “Evaluación de la gobernanza en los espacios naturales protegidos. El caso de la Columbia Británica y Ontario (Canadá)". Anales de Geografía de la Universidad Complutense, 32 (1), 133-151.

Rosenzweig, P.L. (2004). "Informe del Fondo Mexicano para la Conservación de la Naturaleza” [en línea]. Disponible en: http://fmcn.org/wp-content/ uploads/2012/02/FMCN_informe_2004.pdf [2013, 28 de abril].

Sánchez-García, P. et al. (2009). Evaluación de estrategia de cobertura y focalización del Programa de Conservación para el Desarrollo Sostenible (Procodes). Chapingo, México: Centro Regional Universitario del Noroeste/ Universidad Autónoma Chapingo, 132 pp. 
Scheyvens, R. (1999). "Ecotourism and the Empowerment of Local Communities”. Tourism Management, 20, 245-249.

Segrado, R., L. Arroyo y K. Amador (2010). "La zonificación y su aplicación en las Áreas Naturales Protegidas de uso turístico de Quintana Roo”. El Periplo Sustentable, 19, 69-91.

Sosa-Cordero, E., M.L.A. Liceaga-Correa y J.C. Seijo (2008). “The Punta Allen Lobster Fishery: Current Status and Recent Trends”, en R. Townsend, R. Shotton y H. Uchida (eds.). Case Studies in Fisheries Self-Governance. Roma: Organización de las Naciones Unidas para la Agricultura (FAo) (Fisheries Technical Paper, 504).

Vargas, F. (1984). Parques nacionales de México y reservas equivalentes: Pasado, presente y futuro. México: Instituto de Investigaciones EconómicasUNAM.

Varillas, A. (2008). "Defensa de ciudadanos del Parque Kaba”. El Universal, Cancún, 17 de enero. 